\title{
Spontaneous symmetry breaking in fermionic random matrix model
}

\section{Irina Aref'eva and Igor Volovich}

Steklov Mathematical Institute, Russian Academy of Sciences, Gubkina str. 8, Moscow 119991, Russia

E-mail: arefeva@mi-ras.ru, volovich@mi-ras.ru

ABSTRACT: A fermionic random matrix model, which is a 0-dimensional version of the SYK model with replicas, is considered. The replica-off-diagonal correlation functions vanish at finite $N$, but we show that they do not vanish in the large $N$ limit due to spontaneous symmetry breaking. We use the Bogoliubov quasi-averages approach to studying phase transitions. The consideration may be relevant to the study of the problem of existence of the spin glass phase in fermionic models.

KEywords: Spontaneous Symmetry Breaking, 1/N Expansion, Random Systems

ARXIV EPRINT: 1902.09970 


\section{Contents}

1 Introduction 1

2 Setup 2

2.1 Spontaneous symmetry breaking and quasi-averages 2

2.2 Example 3

3 Fermionic random matrix model 3

3.1 Model 3

3.2 Quasi-averages 4

4 Large $N$ asymptotics and quasi-averaging 5

$4.1 q=2$ model 5

$4.2 q=4$ model 8

$\begin{array}{llr}5 & \text { Conclusion } & 9\end{array}$

\section{Introduction}

Spontaneous symmetry breaking is a common occurrence in solid state and high energy physics including the Ising model, Bose-Einstein condensation, Higgs mechanism and spin glasses [1-5]. In these cases the stable solutions of the equations, which govern the system, exhibit less symmetry than the equations themselves. One has a quantum state that is invariant under a symmetry group and which admits a nontrivial decomposition into extremal states corresponding to pure thermodynamic phases. Usually spontaneous symmetry breaking shows in the range of parameters where the phase transitions take place.

In this note a fermionic random matrix model [6-8] is considered which is a 0 -dimensional version of the SYK model [9-11] with a finite number of replicas. The replica-offdiagonal correlations functions vanish at finite $N$, but we show that they do not vanish in the large $N$ limit due to spontaneous symmetry breaking. We use the Bogoliubov quasiaverages approach $[2,12]$ to studying phase transitions.

We consider a model of fermions $\psi_{j}^{\alpha}$ where $j=1, \ldots, N, \alpha=1, \ldots, M$ with the Hamiltonian or action which are invariant under the "gauge group" transformations $\psi_{j}^{\alpha} \rightarrow$ $\epsilon^{\alpha} \psi_{j}^{\alpha}$ where $\epsilon^{\alpha}= \pm 1$. It follows that for finite $N$ the 2-point off-diagonal correlation function vanishes $\left\langle\psi_{j}^{\alpha} \psi_{j}^{\beta}\right\rangle_{N}=0, \alpha \neq \beta$. One can also argue that the 2-point correlation function for finite $N$ vanishes, since it factorizes into fermion 1-point functions which are zero. In details this argument has been considered in [13].

However, we will show that in the fermionic random matrix model in the limit $N \rightarrow \infty$ there is spontaneous symmetry breaking and the 2-point off-diagonal correlation function is 
non-zero. It goes as follows. Let $f_{N}(A), A=\left(A_{\alpha \beta}\right)$ is a generating functional (the density of the free energy) for correlation functions and define the order parameter

$$
Q_{\alpha \beta}^{(N)}=\left.\frac{\partial f_{N}(A)}{\partial A_{\alpha \beta}}\right|_{A=0}=\frac{1}{N} \sum_{j=1}^{N}\left\langle\psi_{j}^{\alpha} \psi_{j}^{\beta}\right\rangle_{N}=0 .
$$

So, if $N$ is finite, the order parameter $Q_{\alpha \beta}^{(N)}=0$. However, if we first compute the large $N$ limit, $\lim _{N \rightarrow \infty} f_{N}(A)=f(A)$ and then compute the derivative,

$$
Q_{\alpha \beta}=\left.\frac{\partial f(A)}{\partial A_{\alpha \beta}}\right|_{A=0},
$$

we will find in this model that the order parameter $Q_{\alpha \beta}$ is nonvanishing. This shows the occurrence of spontaneous symmetry breaking.

Various aspects of the non-diagonal solutions of the saddle point equations and Schwarzian corrections in the SYK and related models are discussed in [6-8, 13-24].

The model considered in this note is a 0-dimensional analogue of the conformal limit of the SYK model in which nondiagonal saddle points have been studied in [19,21] while the model investigated in [7] is an analogue of the total SYK model with replicas.

In the next section spontaneous symmetry breaking and the quasi-averages method are exposed. In section 3 the fermionic matrix model and the order parameter are discussed. In section 4 the cases $q=2$ and $q=4$ are considered and spontaneous breaking of symmetry is shown.

\section{Setup}

\subsection{Spontaneous symmetry breaking and quasi-averages}

A powerful method for investigation of the spontaneous symmetry breaking is the Bogoliubov method of quasi-averages, see [2] and for a recent review [12]. The quasi-averages method proceeds as follows. If, in a finite volume $\Lambda$, one has a Hamiltonian $H$ which is invariant under a symmetry group, then one replaces $H \rightarrow H+w B$ where $B$ is a suitable extensive operator breaking the symmetry and $w$ is a real parameter. Define the partition function $Z_{\Lambda}(w)=\operatorname{Tr} e^{-\beta(H+w B)}, \beta>0$. Now we first take the infinite volume thermodynamic limit $\Lambda \rightarrow \mathbb{R}^{d}$ for the free energy

$$
f(w)=-\lim _{\Lambda \rightarrow \mathbb{R}^{d}} \frac{1}{\beta|\Lambda|} \log Z_{\Lambda}(w)
$$

and for correlation functions and then compute the limit of the free energy at $w=0, f(0)=$ $\lim _{w \rightarrow+0} f(w)$ and the same for its derivatives $f^{\prime}(0), f^{\prime \prime}(0), \ldots$ and for the correlation functions

$$
\prec K \succ=\lim _{w \rightarrow+0} \lim _{\Lambda \rightarrow \mathbb{R}^{d}} \operatorname{Tr}\left(K e^{-\beta(H+w B)}\right) / Z_{\Lambda}(w)
$$

where $K$ is some operator. It may happen that after taking the limit $w \rightarrow 0$ still there is a dependance on $B$ in $f^{\prime}(0), f^{\prime \prime}(0), \ldots$ or in the correlation functions. In such a case one speaks of spontaneous symmetry breaking. In particular, $\prec K \succ$ is called the quasi-average of the operator $K$. The quantity $\prec B \succ$ can be interpreted as an order parameter in the pure phase, selected by the symmetry breaking source. 


\subsection{Example}

There is a simple example of application of quasi-averages for describing spontaneous symmetry breaking. Consider the partition function with the Higgs potential

$$
Z_{N}=\int_{\mathbb{R}} e^{-N\left(x^{2}-a^{2}\right)^{2}} d x
$$

where $a>0, N=1,2,3, \ldots$ There is a symmetry under transformation $x \rightarrow-x,\langle x\rangle_{N}=$ 0 and moreover the free energy vanishes in the large $N$ limit:

$$
f_{0}=-\lim _{N \rightarrow \infty} \frac{1}{N} \log Z_{N}=0 .
$$

Now we add a term breaking the symmetry:

$$
Z_{N}(w)=\int_{\mathbb{R}} e^{-N S} d x, \quad S=\left(x^{2}-a^{2}\right)^{2}+w x, w>0 .
$$

Then the free energy is

$$
f(w)=\lim _{N \rightarrow \infty} f_{N}(w)=-\lim _{N \rightarrow \infty} \frac{1}{N} \log Z_{N}(w)=\left(x_{0}^{2}-a^{2}\right)^{2}+w x_{0},
$$

where $x_{0}=x_{0}(w)$ is a solution of the equation

$$
4 x\left(x^{2}-a^{2}\right)+w=0
$$

with the lowest value of the action $S$. Therefore we obtain

$$
\left.f^{\prime}(w)\right|_{+0}=-a .
$$

So there is a spontaneous symmetry breaking and quasi-average $\prec x \succ=-a$. Note that in this consideration the limit of large $N$ plays a role of the thermodynamical limit.

Uniqueness of the solution $x_{0}=x_{0}(w), w>0$, is used also when one performs the Legendre transform from the free energy $f(w)$ to the effective potential $\Gamma(\phi)=f(w)-w \phi$, $\phi=\partial_{w} f(w)$.

\section{Fermionic random matrix model}

\subsection{Model}

We consider a polynomial function in anticommuting variables of the form [7]

$$
\mathcal{A}=\mathcal{A}_{0}(J)+\mathcal{A}_{1}(A),
$$

where

$$
\begin{aligned}
& \mathcal{A}_{0}(J)=(i)^{q / 2} \sum_{\alpha} \sum_{k_{1}<k_{2}<\ldots<k_{q}} J_{k_{1} k_{2} \ldots k_{q}} \chi_{k_{1}}^{\alpha} \chi_{k_{2}}^{\alpha} \ldots \chi_{k_{q}}^{\alpha}, \\
& \mathcal{A}_{1}(A)=\frac{i}{2} \sum_{\alpha, \beta, j} \chi_{j}^{\alpha} A_{\alpha \beta} \chi_{j}^{\beta} .
\end{aligned}
$$


Here $\chi_{j}^{\alpha}$ are the Grassmann hermitean variables. We consider the zero dimensional model, so the Grassmann variables $\chi_{j}^{\alpha}$ do not depend on time $\tau$. One has $\left\{\chi_{j}^{\alpha}, \chi_{k}^{\beta}\right\}=0, k, j=$ $1, \ldots N, \alpha, \beta=1, \ldots M, q$ is an even natural number, and $\mathbf{J}=\left(J_{k_{1} k_{2} \ldots k_{q}}\right)$ are Gaussian random variables with zero mean $\left\langle J_{k_{1} k_{2} \ldots k_{q}}\right\rangle=0$, and variance is given by $\left\langle J_{k_{1} k_{2} \ldots k_{q}}^{2}\right\rangle=$ $(q-1) ! J^{2} / N^{q-1}, J>0$. The matrix $\left(A_{\alpha \beta}\right)$ is antisymmetric with complex entries. To get correlation functions we will differentiate the generating functional over tensor type source $A_{\alpha \beta}$ and then set $A_{\alpha \beta}=0$.

Although in this note we treat $A_{\alpha \beta}$ as auxiliary parameters in the generating functional, one can interpret them as coupling constants, such models are discussed in 0-dim case in [7] and in 1-dim in [23, 24].

The generating functional (partition function) in the fermionic random matrix model is defined as the Grassmann (Berezin) integral over anticommuting variables

$$
Z_{N}(A)=\int d \mu(\mathbf{J}) \int d \chi e^{i \mathcal{A}}
$$

Here $d \mu(\mathbf{J})$ is a Gaussian probability measure. Mathematical questions of superanalysis are considered in $[25,26]$. The integral over Grassmann variables for $A_{\alpha \beta}=0$ vanishes unless $N$ is a multiple of 4 , so we assume that $N$ is a multiple of 4 . Note also that $Z_{N}(A)$ is a polynomial at $J^{2}$ and $A_{\alpha \beta}$ because there is only a finite number of the Grassmann variables.

The free energy is defined as

$$
f_{N}(A)=-\frac{1}{N} \log Z_{N}(A) .
$$

We are interested in correlation functions

$$
Q_{\alpha \beta}^{(N)}=\left.\frac{\partial}{\partial A_{\alpha, \beta}} f_{N}(A)\right|_{A=0}=\frac{1}{N} \sum_{j=1}^{N}\left\langle\chi_{j}^{\alpha} \chi_{j}^{\beta}\right\rangle_{N} .
$$

Note that $Q_{\alpha \beta}^{(N)}$ vanishes if $N$ is finite,

$$
Q_{\alpha \beta}^{(N)}=0, \quad \alpha \neq \beta
$$

since the 2-point correlation function (3.4) factorizes into fermion 1-point functions, which vanish. Another way to get the relation (3.5) is by notice that the function $\mathcal{A}_{0}(J)$ is invariant under the "gauge transformation" $\chi_{j}^{\alpha} \rightarrow \epsilon_{\alpha} \chi_{j}^{\alpha}$ where $\epsilon_{\alpha}= \pm 1$. The relation (3.5) can be interpreted by saying that there is no symmetry breaking at finite $N$.

\subsection{Quasi-averages}

However if we first take the limit $N \rightarrow \infty$,

$$
f(A)=\lim _{N \rightarrow \infty} f_{N}(A)
$$

and then compute the derivative, we could get a non-zero result for 2-point off-diagonal correlation function defined as

$$
Q_{\alpha \beta}=\left.\frac{\partial f(A)}{\partial A_{\alpha \beta}}\right|_{A=0} .
$$


This is the Bogoliubov quasi-averages approach to phase transitions and spontaneous symmetry breaking. The quantity $Q_{\alpha \beta}$ is called the quasi-average correlation function or the order parameter.

The expression (3.2) can be written also by using the real variables

$$
Z_{N}(A)=\left(\frac{N}{2 \pi}\right)^{\frac{M(M-1)}{2}} \int d \Sigma d G e^{N S}
$$

where

$$
S=\log (\operatorname{Pf}(\mathbf{A}+i \boldsymbol{\Sigma}))+\sum_{\alpha<\beta}\left[-\frac{J^{2}}{q} G_{\alpha \beta}^{q}+i \Sigma_{\alpha \beta} G_{\alpha \beta}\right] .
$$

Here Pf is the Pfaffian, $\mathbf{G}=\left(G_{\alpha \beta}\right)$ and $\boldsymbol{\Sigma}=\left(\Sigma_{\alpha \beta}\right)$ are antisymmetric matrices with real entries, $\mathbf{A}=\left(A_{\alpha \beta}\right)$ is antisymmetric complex matrix, $q$ is an even integer. Note the presence of the factor $\exp \left\{-J^{2} G_{\alpha \beta}^{q} / q\right\}$ which provides the convergence of the integral. Note also that in (2.3) and (3.8) we use the different signs in front of the actions. We will show that for $q=2,4$ and $M=2$ the free energy $f(\mathbf{A})$ in the limit $N \rightarrow \infty$ is equal to the action function $S_{0}$ computed at the saddle point $\left(G_{(0)}, \Sigma_{(0)}\right)$, i.e. $f(\mathbf{A})=-S_{0}(\mathbf{A})$. The saddle point equations read

$$
(\mathbf{A}+i \boldsymbol{\Sigma})^{-1}=-\mathbf{G}, \quad \Sigma_{\alpha \beta}=-i J^{2}\left(G_{\alpha \beta}\right)^{q-1} .
$$

Since the gradient of the action function vanishes at the saddle point we obtain the following expression for the order parameter

$$
Q_{\alpha \beta}=-\left.\frac{\partial S_{0}(\mathbf{A})}{\partial A_{\alpha \beta}}\right|_{A=0}=\left.i\left(\boldsymbol{\Sigma}_{(0)}^{-1}\right)_{\alpha \beta}\right|_{A=0}=\left.G_{(0) \alpha \beta}\right|_{A=0} .
$$

Let us note that the order parameter could depend on the way in which $A \rightarrow 0$ in complex domain. In this note we take $M=2$ and denote $A=A_{12}=a \mathrm{e}^{i \varphi}, a>0$. Then we can define

$$
Q(\varphi)=\left.\mathrm{e}^{-i \varphi} \frac{\partial}{\partial a} f\left(a \mathrm{e}^{i \varphi}\right)\right|_{a=0}
$$

If $Q(\varphi)$ is non-zero then one has spontaneous symmetry breaking.

\section{Large $N$ asymptotics and quasi-averaging}

\section{$4.1 q=2$ model}

We start with $q=2, M=2$ case.

$$
\begin{aligned}
Z_{N}(A) & =\frac{N}{2 \pi} \int_{\mathbb{R}} d y(A+i y)^{N} \int_{\mathbb{R}} d x \exp \left\{N\left[-\frac{J^{2}}{2} x^{2}+i x y\right]\right\} \\
& =\frac{N}{2 \pi} \int d x d y \exp \left\{N\left[-\frac{J^{2}}{2} x^{2}+i x y+\ln (A+i y)\right]\right\}
\end{aligned}
$$


where we use the parametrization

$$
\left(A_{\alpha \beta}\right)=\left(\begin{array}{cc}
0 & A \\
-A & 0
\end{array}\right), \quad\left(G_{\alpha \beta}\right)=\left(\begin{array}{cc}
0 & x \\
-x & 0
\end{array}\right), \quad\left(\Sigma_{\alpha \beta}\right)=\left(\begin{array}{cc}
0 & y \\
-y & 0
\end{array}\right) .
$$

Here $A$ is a complex parameter with small enough $|A|$. The stationary point equations are

$$
\begin{aligned}
-J^{2} x+i y & =0 \\
\frac{i}{A+i y}+i x & =0
\end{aligned}
$$

The solutions to these equations have the form

$$
\begin{aligned}
x_{1} & =\frac{-A-i \sqrt{4 J^{2}-A^{2}}}{2 J^{2}}, \\
y_{1} & =\frac{i}{2}\left(A+i \sqrt{4 J^{2}-A^{2}}\right), \\
x_{2} & =\frac{-A+i \sqrt{4 J^{2}-A^{2}}}{2 J^{2}}, \\
y_{2} & =-\frac{i}{2}\left(-A+i \sqrt{4 J^{2}-A^{2}}\right) .
\end{aligned}
$$

The action at the stationary points reads

$$
\begin{aligned}
& S_{1}(A)=\log \left(\frac{1}{2}\left(A-i \sqrt{4 J^{2}-A^{2}}\right)\right)+\frac{1}{4}\left(\frac{A^{2}}{J^{2}}-2+\frac{i A \sqrt{4 J^{2}-A^{2}}}{J^{2}}\right) \\
& S_{2}(A)=\log \left(\frac{1}{2}\left(A+i \sqrt{4 J^{2}-A^{2}}\right)\right)+\frac{1}{4}\left(\frac{A^{2}}{J^{2}}-2-\frac{i A \sqrt{4 J^{2}-A^{2}}}{J^{2}}\right) .
\end{aligned}
$$

We consider these action for $A=a \mathrm{e}^{i \varphi}, a>0$ and get

$$
\begin{aligned}
& S_{1}(a, \varphi)=\log \left(\frac{1}{2}\left(a \mathrm{e}^{i \varphi}-i \sqrt{4 J^{2}-a^{2} \mathrm{e}^{2 i \varphi}}\right)\right)+\frac{1}{4}\left(\frac{a^{2} \mathrm{e}^{2 i \varphi}}{J^{2}}-2+\frac{i a \mathrm{e}^{i \varphi} \sqrt{4 J^{2}-a^{2} \mathrm{e}^{2 i \varphi}}}{J^{2}}\right) \\
& S_{2}(a, \varphi)=\log \left(\frac{1}{2}\left(a \mathrm{e}^{i \varphi}-i \sqrt{4 J^{2}-a^{2} \mathrm{e}^{2 i \varphi}}\right)\right)+\frac{1}{4}\left(\frac{a^{2} \mathrm{e}^{2 i \varphi}}{J^{2}}-2-\frac{i a \mathrm{e}^{i \varphi} \sqrt{4 J^{2}-a^{2} \mathrm{e}^{2 i \varphi}}}{J^{2}}\right) .
\end{aligned}
$$

In figure 1 we plot the real and imaginary parts of actions (4.11) and (4.12) for $0<$ $a<2$. We see that the real part of the first solution for $0<\varphi<\pi$ is less then the real part of the action on the second one and the second solution dominates. For $\pi<\varphi<2 \pi$ the first solution dominates.

In figure 2 we show the real parts of the actions also for $a>2$. We see the order of dominance is changed for $a>2$. 


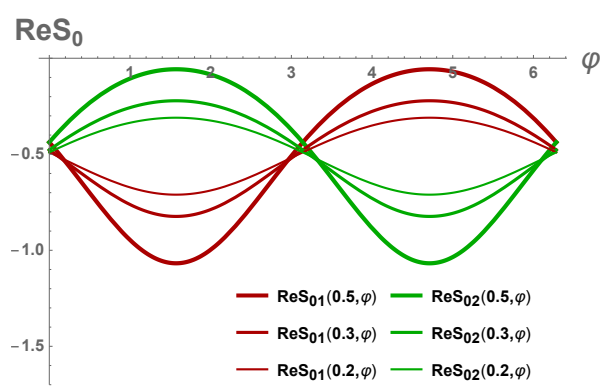

(A)

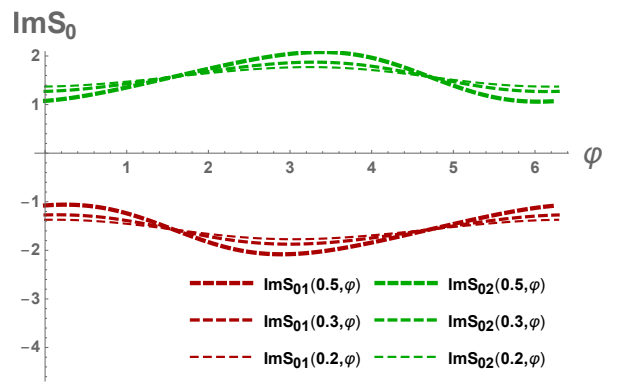

(B)

Figure 1. Plots of real (A) and imaginary (B) parts of the actions on the stationary point, $S_{0, i}(a, \varphi)$ for $J=1$, different $a<2$ and $0<\varphi<2 \pi . i=1,2$ corresponds to the first and second stationary points and are presented by red and green color, respectively.

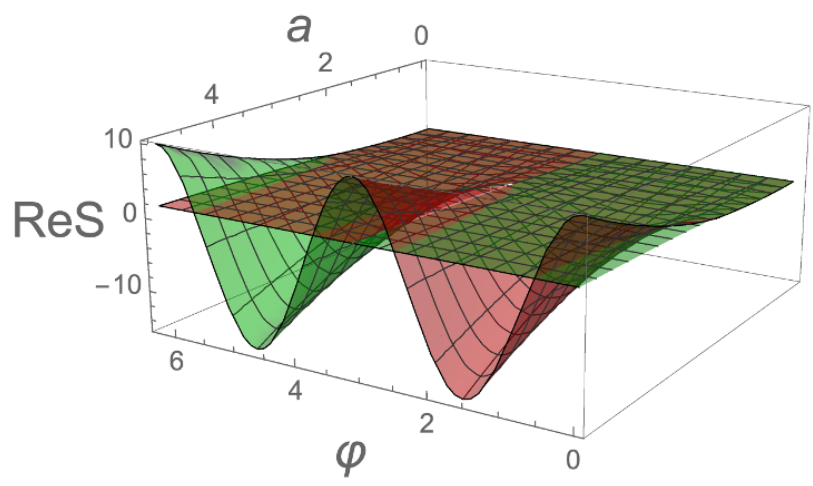

\section{$\operatorname{ReS}_{01}$ \\ $\operatorname{ReS}_{02}$}

Figure 2. Plots for real parts of the actions at the stationary point, $\operatorname{Re} S_{0, i}(a, \varphi)$ for $J=1$, $0<a<4$ and $0<\varphi<2 \pi . i=1,2$ corresponds to the first and second stationary points and are presented by red and green color respectively.

Therefore the free energy for $A=a e^{i \varphi}(0<a<2)$ is given by

$$
f(a, \varphi)=\left\{\begin{array}{l}
-S_{2}(a, \varphi) \text { for } 0<\varphi<\pi \\
-S_{1}(a, \varphi) \text { for } \pi<\varphi<2 \pi
\end{array}\right.
$$

Now we can evaluate the value of the order parameter $Q$. We have

$$
\begin{aligned}
\frac{\partial}{\partial a} S_{1}(a, \varphi) & =\frac{2}{a-i e^{-i \varphi} \sqrt{4 J^{2}-a^{2} e^{2 i \varphi}}} \\
\frac{\partial}{\partial a} S_{2}(a, \varphi) & =\frac{2}{a+i e^{-i \varphi} \sqrt{4 J^{2}-a^{2} e^{2 i \varphi}}} .
\end{aligned}
$$

Now we can obtain the order parameter $Q$. We finally get

$$
Q(\varphi)=- \begin{cases}\left.e^{-i \varphi} \frac{\partial}{\partial a} S_{2}(a, \varphi)\right|_{a=0}=\frac{i}{J}, & 0<\varphi<\pi \\ \left.e^{-i \varphi} \frac{\partial}{\partial a} S_{1}(a, \varphi)\right|_{a=0}=-\frac{i}{J}, & \pi<\varphi<2 \pi .\end{cases}
$$


The last formula shows that there is spontaneous symmetry breaking and the offdiagonal correlation function is nonvanishing.

Note that the partition function (4.1) can be written using the Hermite polynomials [7]

$$
Z_{N}(A)=2\left(\frac{J}{\sqrt{2 N}}\right)^{N} H_{N}\left(\frac{A}{J} \sqrt{\frac{N}{2}}\right) .
$$

The asymptotic behaviour of the Hermite and more general polynomials in the complex domain is evaluated in [27].

\section{$4.2 q=4$ model}

Our starting point is

$$
\begin{aligned}
Z_{N}(A) & =\frac{N}{2 \pi} \int d y \int d x(i y+A)^{N} \exp \left\{N\left(-J^{2} \frac{x^{4}}{4}+i x y\right)\right\} \\
& =\frac{N}{2 \pi} J^{N / 2} \int d y \int d x \exp \{N S\}
\end{aligned}
$$

where

$$
S=-\frac{x^{4}}{4}+i x y+\log (i y+\tilde{A})
$$

and $\tilde{A}=A / \sqrt{J}$.

Here $x, y$ are real variables and $A$ is a complex parameter, $J>0$ and $N$ is a natural number.

The saddle points for $N \rightarrow \infty$ are derived from equations

$$
\begin{gathered}
-x^{3}+i y=0, \\
\frac{1}{\tilde{A}+i y}+x=0 .
\end{gathered}
$$

There are 4 roots and we numerate them by index $i$. The values of the action (4.19) at these roots are denoted by $S_{0 i}, i=1,2,3,4$. The real parts of the action on these roots are degenerate in the sense that they are pairwise equal for real $A$. To remove this degeneracy as in the case $q=2$ we consider the complex $A=a e^{i \varphi}, a>0$. The values of the real parts of the actions are presented in figure 3 for a particular $a=0.1$ and $J=1$. We see that the real part of the fourth solution for $0<\varphi<\pi / 2$ is bigger than the real parts of the action on other solutions. For $\pi / 2<\varphi<\pi$ the first solution dominates, for $\pi<\varphi<3 / 2 \pi$ the second solution dominates and for $3 / 2 \pi<\varphi<2 \pi$ the third one.

The absence of degeneracy for complex $A$ is also shown in the $3 \mathrm{D}$ plot in figure 4 . The plot in figure $4 \mathrm{~B}$ shows that the order of dominance is changed at $a=a_{0}>1$. 


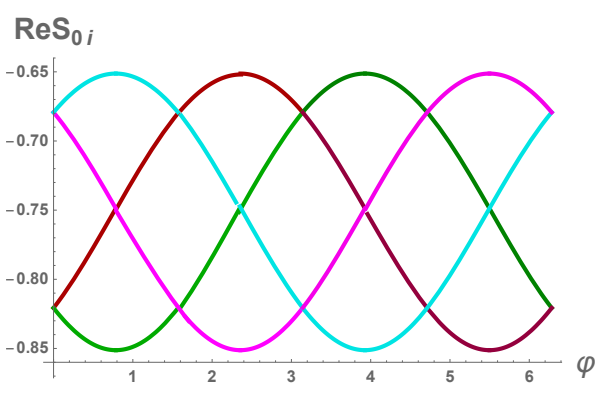

$-\operatorname{ReS}_{01}$

- $\operatorname{ReS}_{02}$

- $\operatorname{ReS}_{03}$

(A)

Figure 3. (A). Plots for real parts of the actions on the stationary point functions, $\operatorname{Re} S_{0, i}(a, \varphi)$ for $J=1, a=0.1$ and $0<\varphi<2 \pi, i=1,2,3,4$ corresponding to the first, second, third and fourth stationary points are presented by darker red, green, magenta and cyan color, respectively.

Calculating the first derivatives of the actions $S_{0, i}(a, \varphi)$ we get

$$
\begin{aligned}
\left.\frac{\partial}{\partial a} S_{0,1}(a, \varphi)\right|_{a=0} & =-\frac{(1-i) e^{i \varphi}}{\sqrt{2}} \\
\left.\frac{\partial}{\partial a} S_{0,2}(a, \varphi)\right|_{a=0} & =-\frac{(1+i) e^{i \varphi}}{\sqrt{2}} \\
\left.\frac{\partial}{\partial a} S_{0,3}(a, \varphi)\right|_{a=0} & =\frac{(1+i) e^{i \varphi}}{\sqrt{2}} \\
\left.\frac{\partial}{\partial a} S_{0,4}(a, \varphi)\right|_{a=0} & =\frac{(1-i) e^{i \varphi}}{\sqrt{2}} .
\end{aligned}
$$

To compute the value of the order parameter we have to take the derivative of the action at the branch with the maximal real part in the considered domain of $\varphi$. Finally we obtain

$$
Q(\varphi)=\left\{\begin{array}{cl}
-\frac{(1-i)}{\sqrt{2}} & \text { for } 0<\varphi<\pi / 2 \\
\frac{(1-i)}{\sqrt{2}} & \text { for } \pi / 2<\varphi<\pi \\
\frac{(1+i)}{\sqrt{2}} & \text { for } \pi<\varphi<3 / 2 \pi \\
-\frac{(1+i)}{\sqrt{2}} & \text { for } 3 \pi / 2<\varphi<2 \pi
\end{array}\right.
$$

Nonvanishing of the order parameter means that there is spontaneous symmetry breaking.

\section{Conclusion}

We have considered the fermionic random matrix model which is a 0 -dimensional version of the SYK model with a finite number of replicas. It is shown that although the 2-point non-diagonal correlation function in the fermionic matrix model vanishes at finite $N$, it does not vanish in the large $N$ limit due to spontaneous symmetry breaking. It would be interesting to study whether this phenomena may occur in other models. 


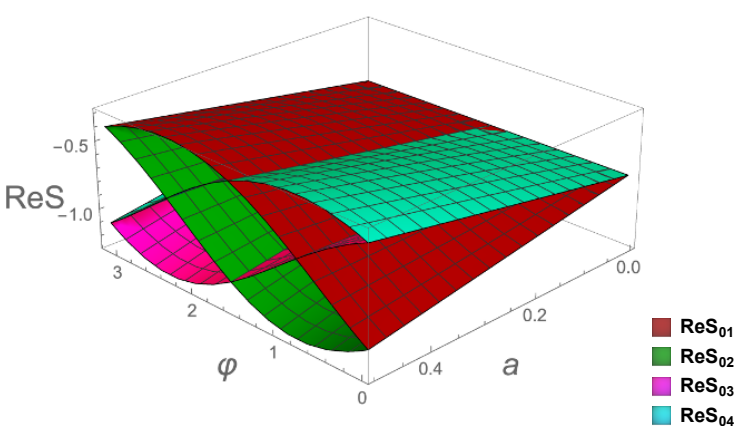

(A)

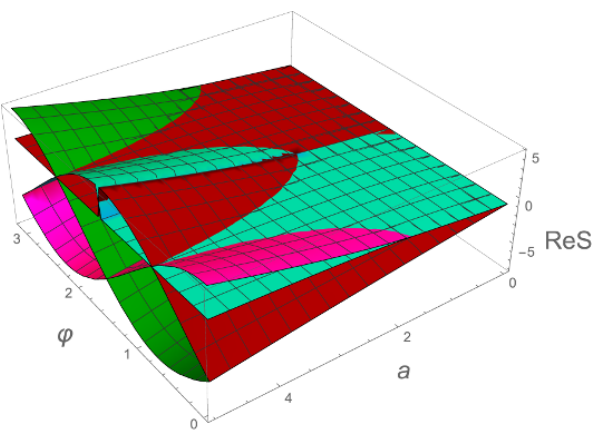

(B)

Figure 4. (A) 3D plots for functions $\operatorname{Re} S_{0,1}\left(a e^{i \varphi}\right)$ (red), $\operatorname{Re} S_{0,2}\left(a e^{i \varphi}\right)$ (green), $\operatorname{Re} S_{0,3}\left(a e^{i \varphi}\right.$ ) (magenta) and $\operatorname{Re} S_{0,4}\left(a e^{i \varphi}\right)$ (cyan) at $J=1$, small $a$ and $0<\varphi<\pi$ show that the complex $A=a e^{i \varphi}$ removes the degeneracy of the real part of the action. (B) The same plot as on (A), but for large variety of $a, 0<a<5$.

\section{Acknowledgments}

We thank M. Khramtsov and V. Zagrebnov for useful discussions. This work is supported by Russian Science Foundation, project 19-11-00320.

Open Access. This article is distributed under the terms of the Creative Commons Attribution License (CC-BY 4.0), which permits any use, distribution and reproduction in any medium, provided the original author(s) and source are credited.

\section{References}

[1] L.D. Landau, On the theory of phase transitions, in Collected Papers. Volume 1, Nauka, Moscow Russia (1969), pp. 234-252 [Zh. Eksp. Teor. Fiz. 7 (1937) 19] [InSPIRE].

[2] N.N. Bogoliubov, Lectures on quantum statistics. Volume 2: Quasi-Averages, Gordon and Breach Science Publishers (1970), republished in Collection of Scientific Works in Twelve Volumes: Statistical Mechanics. Volume 6, Nauka, Moscow Russia (2007).

[3] Y. Nambu, Quasiparticles and Gauge Invariance in the Theory of Superconductivity, Phys. Rev. 117 (1960) 648 [INSPIRE].

[4] S. Weinberg, The quantum theory of fields. Volume 2: Modern applications, Cambridge University Press, Cambridge U.K. (1996).

[5] M. Mezard, G. Parisi and M. Virasoro, Spin Glass Theory and beyond, World Scientific (1987).

[6] J.S. Cotler et al., Black Holes and Random Matrices, JHEP 05 (2017) 118 [Erratum JHEP 09 (2018) 002] [arXiv: 1611.04650] [INSPIRE].

[7] I. Aref'eva and I. Volovich, Notes on the SYK model in real time, Theor. Math. Phys. 197 (2018) 1650 [Teor. Mat. Fiz. 197 (2018) 296] [arXiv:1801.08118] [InSPIRE].

[8] P. Saad, S.H. Shenker and D. Stanford, A semiclassical ramp in SYK and in gravity, arXiv: 1806.06840 [INSPIRE]. 
[9] S. Sachdev and J. Ye, Gapless spin fluid ground state in a random, quantum Heisenberg magnet, Phys. Rev. Lett. 70 (1993) 3339 [cond-mat/9212030] [INSPIRE].

[10] A. Kitaev, A simple model of quantum holography, talk given at the Entanglement in Strongly-Correlated Quantum Matter, Santa Barbara, California, U.S.A., 6 April-2 July 2015 and online at http://online.kitp.ucsb.edu/online/entangled15/.

[11] S. Sachdev, Bekenstein-Hawking Entropy and Strange Metals, Phys. Rev. X 5 (2015) 041025 [arXiv: 1506.05111] [INSPIRE].

[12] W.F. Wreszinski and V.A. Zagrebnov, Bogoliubov quasi-averages: spontaneous symmetry breaking and algebra of fluctuations, Theor. Math. Phys. 194 (2018) 157 [arXiv:1704.00190] [INSPIRE].

[13] G. Gur-Ari, R. Mahajan and A. Vaezi, Does the SYK model have a spin glass phase?, JHEP 11 (2018) 070 [arXiv: 1806.10145] [INSPIRE].

[14] A. Georges, O. Parcollet and S. Sachdev, Quantum fluctuations of a nearly critical Heisenberg spin glass, Phys. Rev. B 63 (2001) 134406 [cond-mat/0009388].

[15] J. Polchinski and V. Rosenhaus, The Spectrum in the Sachdev-Ye-Kitaev Model, JHEP 04 (2016) 001 [arXiv: 1601.06768] [INSPIRE].

[16] W. Fu and S. Sachdev, Numerical study of fermion and boson models with infinite-range random interactions, Phys. Rev. B 94 (2016) 035135 [arXiv:1603.05246] [InSPIRE].

[17] J. Maldacena and D. Stanford, Remarks on the Sachdev-Ye-Kitaev model, Phys. Rev. D 94 (2016) 106002 [arXiv: 1604.07818] [InSPIRE].

[18] A.M. García-García and J.J.M. Verbaarschot, Spectral and thermodynamic properties of the Sachdev-Ye-Kitaev model, Phys. Rev. D 94 (2016) 126010 [arXiv:1610.03816] [InSPIRE].

[19] I. Aref'eva, M. Khramtsov, M. Tikhanovskaya and I. Volovich, Replica-nondiagonal solutions in the SYK model, JHEP 07 (2019) 113 [arXiv:1811.04831] [INSPIRE].

[20] V.V. Belokurov and E.T. Shavgulidze, Simple rules of functional integration in the Schwarzian theory: SYK correlators, arXiv:1811.11863 [INSPIRE].

[21] H. Wang, D. Bagrets, A.L. Chudnovskiy and A. Kamenev, On the replica structure of Sachdev-Ye-Kitaev model, arXiv: 1812.02666 [INSPIRE].

[22] J. Kim, I.R. Klebanov, G. Tarnopolsky and W. Zhao, Symmetry Breaking in Coupled SYK or Tensor Models, Phys. Rev. X 9 (2019) 021043 [arXiv: 1902.02287] [InSPIRE].

[23] J. Maldacena and X.-L. Qi, Eternal traversable wormhole, arXiv:1804.00491 [INSPIRE].

[24] A.M. García-García, T. Nosaka, D. Rosa and J.J.M. Verbaarschot, Quantum chaos transition in a two-site Sachdev-Ye-Kitaev model dual to an eternal traversable wormhole, Phys. Rev. D 100 (2019) 026002 [arXiv:1901.06031] [INSPIRE].

[25] V.S. Vladimirov and I. Volovich, Superanalysis. I. Differential Calculus, Theor. Math. Phys. 59 (1984) 317 [INSPIRE].

[26] V.S. Vladimirov and I. Volovich, Superanalysis. II. Integral calculus, Theor. Math. Phys. 60 (1984) 743.

[27] P. Deift, T. Kriecherbauer, K.T-R. Mclaughlin, S. Venakides and X. Zhou, Strong Asymptotics of Orthogonal Polynomials with Respect to Exponential Weights, Commun. Pure Appl. Math. 52 (1999) 1491. 